\title{
Níveis de ansiedade em cuidadores informais de pacientes internados em domicílios
}

\author{
Anxiety levels in informal caregivers of patients admitted to households \\ Niveles de ansiedad en cuidadores informales de pacientes ingresados en hogares
}

Recebido: 19/07/2021 | Revisado: 27/07/2021 | Aceito: 21/10/2021 | Publicado: 23/10/2021

\author{
Lucélia Campelo de Melo Santos \\ ORCID: https://orcid.org/0000-0001-6087-2765 \\ Universidade Brasil, Brasil \\ E-mail: lucellyacampello@yahoo.com.br \\ Drauzio Eduardo Naretto Rangel \\ ORCID: https://orcid.org/0000-0001-7188-100X \\ Universidade Brasil, Brasil \\ E-mail: drauzio.rangel@universidadebrasil.edu.br
}

\begin{abstract}
Resumo
Objetivo: avaliar a ansiedade em cuidadores informais de pacientes internados em domicílio. Metodologia: Refere-se a um estudo exploratório quantitativo, realizado com 49 cuidadores informais de pacientes internados em domicílio. A coleta de dados ocorreu com os instrumentos: questionário sociodemográfico e de estilo de vida e escala de avaliação ansiedade de Hamilton. A pesquisa foi aprovada pelo Comitê de Ética e Pesquisa da Universidade Brasil. Resultados: Os perfis dos cuidadores são em sua maioria do sexo feminino, sendo filhas e esposas, em sua maioria com nível de escolaridade superior incompleto, dos investigados $(65,30 \%)$ apresentaram uma condição de ansiedade normal, mesmo em uma parcela pequena de cuidadores com ansiedade moderada (12,24\%), um dos fatores relatados foi a sobrecarga de tarefas centrada em um indivíduo, denominado de cuidador pode acarretar um processo de adoecimento físico e psicológico. Conclusões: Os dados obtidos no estudo foram contrários à maioria das pesquisas, que nas amostras estudadas apresentam o maior percentual de indivíduos avaliados com scores compatíveis com a ansiedade patológica.
\end{abstract}

Palavras-chave: Ansiedade; Hospitalização; Cuidadores informais.

\begin{abstract}
Home care is a model in which the maintenance of the patient's health requires the care of a caregiver, who dedicates part of their day and routine to care work, thus, these caregivers are subjected to physical and psychological wear and tear, which can lead to high levels of anxiety, this way the work aims to assess anxiety in informal caregivers of patients hospitalized at home. It refers to a non-randomized clinical trial carried out with 49 informal caregivers of patients hospitalized at home. Data collection was carried out using the following instruments: sociodemographic and lifestyle questionnaire and Hamilton's anxiety assessment scale. The research was approved by the Ethics and Research Committee of Universidade Brasil. The profiles of caregivers are mostly female, being daughters and spurs, mostly with incomplete higher education level, of those investigated $(65.30 \%)$ had a normal anxiety condition. The data obtained in the study were contrary to the vast majority of researches, which in the studied samples present the highest percentage of individuals evaluated with scores compatible with pathological anxiety.
\end{abstract}

Keywords: Anxiety; Hospitalization; Informal caregivers.

\section{Resumen}

La atención domiciliaria es un modelo en el que el mantenimiento de la salud del paciente requiere del cuidado de un cuidador, quien dedica parte de su día y rutina en la labor asistencial, por lo que estos cuidadores están sometidos a un esfuerzo físico y psicológico, que puede llevar a niveles elevados. de ansiedad, de esta forma el trabajo tiene como objetivo evaluar la ansiedad en cuidadores informales de pacientes hospitalizados en el domicilio. Se refiere a un ensayo clínico no aleatorizado realizado con 49 cuidadores informales de pacientes hospitalizados en su domicilio. La recogida de datos se realizó mediante los siguientes instrumentos: cuestionario sociodemográfico y de estilo de vida y escala de valoración de ansiedad de Hamilton. La investigación fue aprobada por el Comité de Ética e Investigación de la Universidade Brasil. Los perfiles de los cuidadores son en su mayoría mujeres, siendo hijas y espuelas, en su mayoría con nivel educativo superior incompleto, de los investigados $(65,30 \%)$ presentaban un estado de ansiedad normal. Los datos obtenidos en el estudio fueron contrarios a la gran mayoría de estudios, que en las muestras estudiadas presentan el mayor porcentaje de individuos evaluados con puntuaciones compatibles con ansiedad patológica.

Palabras clave: Ansiedad; Hospitalización; Cuidadores informales. 


\section{Introdução}

A Internação domiciliar (ID) é uma modalidade de atenção à saúde que busca novas formas de promoção de cuidados e de atuação multidisciplinar, que vem aumentando em ampla escala no Brasil e no mundo. A ID busca uma alternativa à internação hospitalar, reduzindo contaminações hospitalares (de Lapena et al., 2011), reduzindo as consequências de um "ciclo vicioso" de doença-hospitalização-dependência que afeta tanto o paciente em sua condição física quanto emocional, como pode afetar também a saúde dos seus familiares e meio social, contribuindo para sua multimorbidade, com elevados custos econômicos e afetivos (Andrade et al., 2017; Sanchis-Soler et al., 2021).

A intervenções no ambiente domiciliar requer uma atenção profissional qualificada, pois alguns dos cuidados que devem ser desenvolvidos necessitam de competências específicas como o profissional de enfermagem, onde tem por função a coordenação do plano de cuidados, ações educacionais e orientação de cuidadores e/ou familiares e ações assistenciais (Minayo, 2019; Silva et al., 2014).

A gestão do cuidado deve envolver múltiplas dimensões, ajustando-se a cada fase da vida buscando a melhor condição de vida para o paciente. $\mathrm{O}$ ambiente domiciliar se apresenta como um cenário propício para o cuidado, com vista a reduzir o sofrimento, pois o paciente se encontra em ambiente mais acolhedor e confortável (Oliveira et al., 2017).

O desenvolvimento de cuidados no ambiente domiciliar permite ao paciente a possibilidade de continuar em seu contexto familiar e social, com atenção multiprofissional especializada e disposta a oferecer, tanto um suporte ao paciente quando aos familiares ou cuidadores, com estes cuidados podem ser evitadas internações recorrentes e desnecessárias o que contribui para uma melhor qualidade de vida do paciente (Faller et al., 2016).

O cuidador é uma peça fundamental na atenção domiciliar, que pode ou não ter um vínculo familiar com o paciente, podendo ser remunerado ou não. Sua função é acompanhar e auxiliar a pessoa a se cuidar, fazendo somente as atividades que ela não consiga fazer sozinha, não incluindo procedimentos da área de enfermagem ou fisioterapia (Boaventura et al., 2016).

O cuidador está exposto a diversas situações estressantes, que devido muitas vezes a uma jornada diária incessante, repetitiva e desgastante, que envolvem muitas vezes as atividades domésticas e cuidados contínuos. Nesta rotina costumeiramente não recebem informações adequadas e nem um apoio psicossocial (Cesário et al., 2017).

As longas rotinas desempenhadas pelos cuidadores podem levá-los a uma sobrecarga, as quais é capaz de comprometer a saúde também deste cuidador, se apresentando diferentes sendo, consequências físicas, psicológicas, sociais e financeiras, por vezes mais ou menos explícitas. Os cuidadores estão envolvidos com os cuidados que muitas vezes abdica de seus cuidados pessoais em detrimento do outro (Cruz et al., 2009; Stachfleth et al.,2012).

O estresse vivenciado por pessoas que exercem a função de cuidador, levam estes a níveis mais elevados de depressão, estresse, ansiedade e outros problemas de saúde, com a progressão da doença tende a se tornar mais graves. O indivíduo que apresenta ansiedade apresenta sentimentos indesejados, dificuldades como irritabilidade, incômodo na realização das tarefas assistenciais e nas relações familiares (Valentini et al. 2010, Dourado et al, 2018).

A ansiedade é um dos transtornos mentais mais comum na população em geral apresentando grande incidência ao longo da vida, além de ser um gatilho para diversas outras patologia sendo um transtorno multifatorial, com maior incidência em mulheres. Ressalta-se que pode ser desencadeada ou mesmo exacerbada por conflitos familiares, desequilíbrio no ciclo circadiano, preocupações excessivas, sedentarismo, comprometendo a saúde do indivíduo acometido por ela (Paula et al., 2017).

Desta forma, a pesquisa tem como objetivo avaliar os níveis de ansiedade em cuidadores informais internados em domicílio na cidade de Teresina-PI. 


\section{Metodologia}

A natureza da pesquisa é quantitativa, sendo que o uso dessa abordagem proporciona o aprofundamento da investigação das questões relacionadas ao fenômeno. Quando a pesquisa qualitativa é associada ao emprego da quantificação, tanto nas modalidades de coleta de informações quanto no tratamento delas por meio de técnicas estatísticas, favorece a compreensão dos fenômenos e cenários (Gil, 1999).

A pesquisa utilizou a estratégia do levantamento de dados, ou seja, a obtenção de dados e informações sobre características, ações ou opiniões de determinado grupo de pessoas, que representa uma população-alvo, por meio de um instrumento de pesquisa, normalmente um questionário (Freitas et al 2000).

\section{Amostragem}

A amostra para o estudo foi composta por cuidadores informais contando com a participação de 49 cuidadores. Como critério de participação o respondente deveria ser o cuidador principal exercer os cuidados a no mínimo 30 dias. Os participantes foram contactados e selecionados por idade mínima e tempo de cuidado. Foram excluídos participantes que não responderam ao questionário de maneira completa.

O presente trabalho foi aprovado pelo Comitê de Ética e Pesquisa da Universidade Brasil, com o parecer n ${ }^{\circ} 4472827$. Os aspectos éticos seguiram as normas estabelecidas na Resolução 466/12 do Conselho Nacional de Saúde foram garantidos por meio do Termo de Consentimento Livre e Esclarecido, atendendo às exigências éticas e científicas fundamentais de uma pesquisa envolvendo seres humanos, onde foram explicados aos participantes por meio dos formulários, as etapas da pesquisa e objetivos da mesma.

\section{Procedimentos.}

Os protocolos para obtenção de dados ocorreram no período de março e abril de 2021, os instrumentos para a coleta de dados foram os formulários contendo características sociodemográficas, contemplando as seguintes variáveis, sexo, idade, escolaridade, grau de parentesco, estado civil, religião, quantidade de moradores na residência, situação de trabalho e renda familiar e a Escala de Avaliação de Ansiedade de Hamilton, a escala de Max Hamilton et al foi desenvolvido na década de 1960, no Brasil. A escala foi validada por Moreno e Moreno (1998).

\section{Resultados e Discussão}

Participaram do estudo, 49 cuidadores informais de pacientes internados em domić́lio, sendo 45 cuidadores do sexo feminino e 4 cuidadores do sexo masculino. A média de idade foi de 49 anos (Mediana: 23,00; Desvio Padrão: 9,174; Mínimo: 18; Máximo: 55). A Tabela 1 apresenta a caracterização social e demográfica dos participantes de estudo. 
Research, Society and Development, v. 10, n. 14, e11101418445, 2021

(CC BY 4.0) | ISSN 2525-3409 | DOI: http://dx.doi.org/10.33448/rsd-v10i14.18445

Tabela 1. Caracterização social e demográfica dos cuidadores informais. Teresina, Piaui.

\begin{tabular}{|c|c|c|}
\hline Indicador & $\mathbf{N}$ & frequência \\
\hline IDADE & $\begin{array}{l}\text { (média } \\
\text { /desvio } \\
\text { padrão) }\end{array}$ & $45.61( \pm 14.4)$ \\
\hline
\end{tabular}

ESTADO CIVIL

$\begin{array}{lcc}\text { Solteira (o) } & 19 & 39 \% \\ \text { Casada(o) } & 26 & 53 \% \\ \text { Divorciada(o) } & 2 & 4 \% \\ \text { Viúvo(a) } & 1 & 2 \%\end{array}$

SITUAÇÃO DE

TRABALHO

$\begin{array}{lcc}\text { Nunca trabalhou } & 9 & 18 \% \\ \text { Trabalha, mas não está em atividade } & 15 & 31 \% \\ \text { Já trabalhou, não trabalha mais } & 10 & 20 \% \\ \text { Trabalha e em atividade no momento } & 15 & 31 \% \\ \text { Aposentado(a) } & 0 & 0 \%\end{array}$

RELIGIÃO

$\begin{array}{lcc}\text { Católico } & 33 & 67 \% \\ \text { Evangélico } & 15 & 31 \% \\ \text { Não possui } & 1 & 2 \%\end{array}$

GRAU DE

PARENTESCO

$\begin{array}{lcc}\text { Filha } & 21 & 43 \% \\ \text { Mãe } & 9 & 18 \% \\ \text { Esposa } & 5 & 10 \% \\ \text { Sobrinha } & 1 & 2 \% \\ \text { Neta } & 1 & 2 \% \\ \text { Cuidadora/vizinha } & 3 & 6 \% \\ \text { Genro/nora } & 3 & 6 \% \\ \text { Tio(a) } & 1 & 2 \% \\ \text { Irmã(o) } & 4 & 8 \% \\ \text { Sem resposta } & 1 & 2 \%\end{array}$

ESCOLARIDADE

$\begin{array}{lcc}\text { Analfabeto } & 1 & 2 \% \\ \text { Fundamental incompleto } & 12 & 24 \% \\ \text { Fundamental completo } & 0 & 0 \% \\ \text { Médio incompleto } & 10 & 20 \% \\ \text { Médio completo } & 0 & 0 \% \\ \text { Superior incompleto } & 17 & 35 \% \\ \text { Superior completo } & 10 & 20 \%\end{array}$

Fonte: Autores.

Para o uso do instrumento Escala de Avaliação de Ansiedade de Hamilton (Moreno \& Moreno, 1988), os resultados mostram que apresentaram ansiedade normal 65,3\%, os que apresentaram reação patológica ligeira 20,40\%, ansiedade 
patológica moderada $12,34 \%$ e para ansiedade patológica grave 2,04\%.

Tabela 2. Apresentação do resultado da Escala de Avaliação de Ansiedade de Hamilton.

\begin{tabular}{lll}
\hline Variável & N & Frequência \\
\hline Resultado Hamilton & 32 & $65,30 \%$ \\
Ansiedade Normal & 10 & $20,40 \%$ \\
Reação Patológica Ligeira & 6 & $12,24 \%$ \\
Ansiedade Patológica & & \\
Moderada & 1 & $02,04 \%$ \\
Ansiedade Patológica Grave & & \\
\hline
\end{tabular}

A caracterização social e demográfica dos cuidadores informais de pacientes em internação domiciliar com idade média geral de 49 anos de idade, sendo a sua maioria do sexo feminino. Os dados corroboram com a pesquisa realizada por Santos e Gutierrez (2013) que apontou que $82 \%$ dos cuidadores informais eram do sexo feminino, com média de idade de 58,3 anos. Dados que revelam que há predominância de cuidadores informais do sexo feminino e com idade superior a 40 anos.

No que concerne ao grau de parentesco (tabela 01), a amostra apresentou a seguinte composição: filhas $(21=43 \%)$, mães $(9=18 \%)$, esposa $(5=10 \%)$, sobrinha $(1=2 \%)$, neta $(1=2 \%)$, vizinhas $(3=6 \%)$, genros/noras $(3=6 \%)$, tio(a) $(1=2$ $\%)$, irmão $(4=8 \%)$ e sem resposta $(1=2 \%)$.

Conforme o estado civil obteve-se a seguinte composição: solteiros $(19=39 \%)$, casados $(26=53 \%)$, divorciados $(2$ $=4 \%)$, e viúvo $(1=2 \%)$.

Os dados reafirmam que os cuidados informais são exercidos majoritariamente por mulheres, que exercem múltiplos papeis, que se sobrepõem ao ato de cuidar de uma paciente em internação domiciliar, essa predominância do sexo feminino como cuidadores, é ainda um perfil histórico onde as responsabilidades dos cuidados aos doentes, é destinado as mulheres. Essas mulheres que em sua maioria apresentam uma sobrecarga de trabalho que também incluem serviços domésticos, cuidados com os filhos, dentre outros (Minayo, 2021; Queiroz et al., 2018; Santos \& Gutierres, 2013).

Os cuidados informais de pacientes em internação domiciliar são executados em sua maioria, por integrantes da família primária: esposa, filhos, filhas, realizado prioritariamente, neste estudo, pela filha. Souza et al., (2015) discorrem que o cuidado domiciliar é um ato complexo, exercido prioritariamente por mulheres, que devem alinhar o ato de cuidar de um indivíduo doente com os cuidados com o lar, preparação de alimentos, ministração de medicações, dentre outras atividades. O cuidador é um sujeito que vivência uma sobrecarga de atividades, no entanto, o contexto, a sua adequação às tarefas, as experiências individuais, influenciarão na sua adaptação ao ato de cuidar. A sobrecarga de tarefas centrada em um indivíduo, denominado de cuidador pode acarretar um processo de adoecimento físico e psicológico.

Em relação às atividades laborais (tabela 1) os dados coletados apontaram que 9 (18\%) nunca exerceu atividades de trabalho formal, 15 (31\%) tem profissão, mas não está em atividade, 25 (51\%) já exerceu trabalho remunerado, mas atualmente não exerce e 15 (31\%) exerce atividades remuneradas. Dados corroboram com o estudo realizado por Souza et al., (2015) que apontou que o os cuidadores principais apresentam longas jornadas de trabalho voltadas ao cuidado do indivíduo doente em domicílio, se abstendo em sua maioria, de atividades laborais fora do ambiente domiciliar

Queiroz et al (2018), verifica que os cuidadores mais jovens apresentam escores da qualidade de vida mais baixo, o principal motivo está relacionado diretamente a vida pessoal e a profissional, visto que os mais jovens apresentam necessidades profissionais e sociais diferentes de pessoas idosas. 
Os cuidados em saúde a outrem é uma tarela permeada de desafios e dificuldades, por isso o cuidador principal necessita de colaboração de outros membros do arranjo familiar ou do ambiente externo na efetivação dos cuidados. O cuidador necessita de apoio social, uma rede de solidariedade, com vistas a melhoria da sua qualidade de vida, diante da tarefa de cuidar (Brandoni, 2010; Marques; Teixeira \& Sousa, 2012).

No que concerne a religião, $33(67 \%)$ afirmaram ser católicos, $15(31 \%)$ evangélicos e 1 (2\%) não informaram possuir uma religião. Pelo exposto, evidencia-se um maior percentual de cuidadores que adotam determinada religião. Para Brondani et. al (2010) a adoção de uma religião proporciona vivências, como meditações, orações que permitem aliviar o sofrimento, em que o cuidador se apoia em um sistema de crenças. Fé de que um ser superior é basilar para o enfrentamento de todos os desafios imbuídos no ato de cuidar.

Os dados resultantes da aplicação da escala de Hamilton (tabela 2) apontaram que 32 (65\%) dos participantes do estudo apresentaram níveis de ansiedade dentro dos padrões de normalidade, 10 (20,4\%) reação patológica ligeira, 6 (12,2\%) apresentaram ansiedade patológica e $1(2,0 \%)$ apresentou ansiedade patológica grave. Os dados apresentados são contrários a achados sobre estados em ansiedade em cuidadores Santos e Gutierrez (2013), em estudo evidenciaram que 80\% da sua amostra apresentou em avaliação, traços compatíveis com ansiedade.

Apesar de ser um recurso adaptativo que acompanha o homem ao longo da sua evolução, a ansiedade em alto grau é compreendida como um transtorno psicológico, comprometendo o organismo em seu quadro de saúde. Além de desencadear uma diversidade de respostas psicofisiológicas, é gatilho para a manifestação de outras patologias físicas e orgânicas (LEAHY, 2011). Assim, é de suma importância a investigação do sofrimento psicológico diante do binômio saúde $\mathrm{x}$ doença.

É de suma importância se pensar na qualidade de vida das cuidadoras, que soma os cuidados informais de saúde a todas as outras funções desempenhadas no lar. No âmbito dos cuidados em saúde pelo Estado, a saúde do cuidador informal tem sido negligenciada, uma vez que não existem ações ou iniciativas voltadas especificamente para esse público, pelo exposto, urge a necessidade do planejamento e execução de ações/políticas que contemplem a saúde do cuidador, diante do ato de cuidar (Minayo, 2021).

O cuidador lida diariamente com uma séria de dificuldades, dentre eles a não valorização do seu trabalho, a escassez ou ausência de equipamentos e formação que possibilitem o e/ou melhorem o ato de cuidar, o sofrimento do outro pela dor da perda da saúde, uma desestruturação na sua rotina, que é preenchida com ações de cuidados ao outro. No Brasil, um dos fatores que contribui para a internação domiciliar é a redução de custos com a saúde, em grandes partes dos casos, mesmo os familiares de pacientes em cuidados paliativos não recebem apoio de maneira correta, pois os cuidadores não possuem conhecimento sobre a doença e sua evolução (Maronesi et at. 2014; Valeberg \& Grov, 2013).

O cuidar é uma ação singular e na figura do cuidador incide um sujeito que proverá em uma série de necessidades do indivíduo que está doente, que vão desde cuidados físicos e de saúde, cuidados psicológicos, como processo de escuta, encorajamento, afeto, até o intermédio de momentos de lazer, como passeio e uso de criatividade para planejar e executar momentos de entretenimento, as atividades psicoeducativas podem contribuir na redução do estresse, ansiedade e sintomas depressivos em cuidadores (Barbora et al., 2012; Brondani et al, 2010).

$\mathrm{O}$ ato de cuidar desencadeia na vida do indivíduo que cuida, vivências conflituosas, permeadas por sobrecarga de trabalho, o que indubitavelmente contribui para quadros de adoecimento. Assim, compete aos profissionais de saúde a proposição e implantação de políticas de saúde que proporcionem suporte aos familiares do paciente em adoecimento, em especial aos seus cuidadores, considerando que a atividade de cuidar é desgastante e implica em riscos para a saúde (Rocha, Vieira \& Sena, 2008). 


\section{Conclusão}

A ansiedade é um transtorno psicológico comum em cuidadores informais de pacientes em internação domiciliar, que afeta a saúde daquele que exerce o ato de cuidar, de forma holística nas perspectivas físicas, psicológicas e sociais. Os dados obtidos no estudo foram contrários à maioria das pesquisas, que nas amostras estudadas apresentam o maior percentual de indivíduos avaliados com scores compatíveis com a ansiedade patológica.

Apesar dos baixos escores compatíveis com transtornos ansiosos, foram evidenciados participantes com resultados compatíveis com ansiedade grave, concomitante com perfis sociodemográficos compatíveis com diversos estudos que apontam que o ato de cuidar em saúde, em domicílio, centra-se em figuras femininas, com sobrecarga de tarefas e que em sua maioria não exercem atividades laborais fora do ambiente domiciliar.

Neste ínterim, é de suma importância se pensar na saúde do cuidador, que não dispõem de ações no âmbito das políticas de saúde, voltadas para as suas necessidades. São indivíduos com ações de cuidados em saúde, em sua maioria, sem preparo técnico, teórico e com sobrecarga de papeis em domicílio.

É valido ressaltar que o estudo apresenta limitações, em relação ao tamanho da amostra, o que dificulta generalizar os achados para todos os cuidadores informais de pacientes em internação domiciliar do cenário nacional. Pelo exposto sugere-se a realização de mais estudos sobre a manifestação da ansiedade em cuidadores informais de pacientes em internação domiciliar.

Com base nas informações coletadas os dados podem servir como base em pesquisa futuras sobre os níveis de ansiedade em cuidadores, as pesquisas futuras podem ser aplicadas de cuidadores em outras regiões, assim como a possibilidade de abordagens metodológicas diferentes.

\section{Agradecimentos}

Esta pesquisa foi apoiada por bolsa do Conselho Nacional de Desenvolvimento Científico e Tecnológico (CNPq) do Brasil PQ1D 302100 / 2018-0 para D.E.N.R

\section{Referências}

Andrade, A. M., Silva, K. L., Seixas, C. T., \& Braga, P. P. (2017). Atuação do enfermeiro na atenção domiciliar: uma revisão integrativa da literatura. Revista Brasileira de Enfermagem, 70, 210-219.

Barbosa, A. L., Cruz, J., Figueiredo, D., Marques, A., \& Sousa, L. (2011). Cuidar de idosos com demência em instituições: competências, dificuldades e necessidades percepcionadas pelos cuidadores formais. Psicologia, Saúde e Doenças, 12(1), 119-129.

Boaventura, L. C., Borges, H. C., \& Ozaki, A. H. (2016). Avaliação da sobrecarga do cuidador de pacientes neurológicos cadeirantes adultos. Ciência \& Saúde Coletiva, 21, 3193-3202.

Brondani, C. M., Beuter, M., Alvim, N. A. T., Szareski, C., \& Rocha, L. S. (2010). Cuidadores e estratégias no cuidado ao doente na internação domiciliar. Texto \& Contexto-Enfermagem, 19, 504-510.

Cesário, V. A. C., Leal, M. C. C., Marques, A. P. D. O., \& Claudino, K. A. (2017). Estresse e qualidade de vida do cuidador familiar de idoso portador da doença de Alzheimer. Saúde em Debate, 41, 171-182.

Cruz, M. N., Lecheta, D. R., \& Wachholz, P. A. (2009). Fatores associados à sobrecarga e à depressão em cuidadores de idosos com doença de Alzheimer. Geriatria \& Gerontologia, 3(1), 15-23.

Dourado, D. M., Rolim, J. A., de Souza Ahnerth, N. M., Gonzaga, N. M., \& Batista, E. C. (2018). Ansiedade e depressão em cuidador familiar de pessoa com transtorno mental. ECOS-Estudos Contemporâneos da Subjetividade, 8(1), 153-167.

Freitas, H., Oliveira, M., Saccol, A. Z., \& Moscarola, J. (2000). O método de pesquisa survey. Revista de Administração da Universidade de São Paulo, 35(3).

GIL, A. C. Métodos e técnicas de pesquisa social. São Paulo: Atlas. 1999.

Leahy, R. L. (2011). Livre de Ansiedade. (V. Figueira, trad.). Porto Alegre: Artmed. 
Research, Society and Development, v. 10, n. 14, e11101418445, 2021

(CC BY 4.0) | ISSN 2525-3409 | DOI: http://dx.doi.org/10.33448/rsd-v10i14.18445

Maronesi, L. C., da Silva, N. R., de Oliveira Cantu, S., \& dos Santos, A. R. (2014). Indicadores de estresse e sobrecarga em cuidadores formais e informais de pacientes oncológicos. Estudos e Pesquisas em Psicologia, 14(3), 877-892.

Marques, M. J. F., Teixeira, H. J. C., \& Souza, D. C. D. B. N. D. (2012). Cuidadoras informais de Portugal: vivências do cuidar de idosos. Trabalho, Educação e Saúde, 10, 147-159.

Moreno R A, \& Moreno D H. Escalas de depressão de Montgomery \& Asberg (MADRS) e de Hamilton (HAM-D). Revista Psiquiatria Clínica. 1998; 25:26272

Minayo M C S. O imperativo de cuidar da pessoa idosa dependente. Cien Saúde Colet. 2019; 24(1):247-252.

Minayo, M. C. D. S. (2021). Cuidar de quem cuida de idosos dependentes: Por uma política necessária e urgente. Ciência \& Saúde Coletiva, $26,7-15$.

Oliveira, M. D. B. P. D., Souza, N. R. D., Bushatsky, M., Dâmaso, B. F. R., Bezerra, D. M., \& Brito, J. A. D. (2017). Oncological homecare: family and caregiver perception of palliative care. Escola Anna Nery, 21.

Paula, F. M., Silvério, G. B., de Araújo Melo, L., Felício, P. V. P., Jorge, R. P. C., \& Da Silva, A. M. (2017). Prevalência e fatores associados à depressão e ansiedade em estudantes de medicina. Rev. Educ. Saúde, 5.

Queiroz, R. S. D., Camacho, A. C. L. F., Gurgel, J. L., Assis, C. R. D. C. D., Santos, L. M. D., \& Santos, M. L. S. C. D. (2018). Sociodemographic profile and quality of life of caregivers of elderly people with dementia. Revista Brasileira de Geriatria e Gerontologia, 21, $205-214$.

Rezende, V. L., Derchain, S. F. M., Botega, N. J., Sarian, L. O., Vial, D. L., \& Morais, S. S. (2005). Depressão e ansiedade nos cuidadores de mulheres em fase terminal de câncer de mama e ginecológico. Revista Brasileira de Ginecologia e Obstetrícia, 27, 737-743.

Rocha, M. P. F., Vieira, M. A., \& Sena, R. R. D. (2008). Desvelando o cotidiano dos cuidadores informais de idosos. Revista Brasileira de Enfermagem, 61, 801-808.

Sadock, B. J., Sadock, V. A., \& Ruiz, P. (2016). Compêndio de Psiquiatria-: Ciência do Comportamento e Psiquiatria Clínica. Artmed Editora.

Sanchis-Soler, G., San Inocencio-Cuenca, D., Llorens-Soriano, P., \& Blasco-Lafarga, C. (2021). Reducción de la sobrecarga del cuidador tras entrenamiento supervisado en ancianos pluripatológicos y paliativos. Cuadernos de Psicología del Deporte, 21(1), 271-281.

Santos, C. F. D., \& Gutierrez, B. A. O. (2013). Avaliação da qualidade de vida de cuidadores informais de idosos portadores da doença de Alzheimer. Revista Mineira de Enfermagem, 17(4), 792-805.

Silva, L. K., Sena, R. R., Silva, P. M., Souza, C. G., \& Martins, A. C. S. (2014). The nurse's role in home care: its implications for the traning process. Cienc. Cuid Saude [Internet], 13(3), 503-10.

Sousa, L. R. Hanus, J. S., Libera L. B. D., Silva, V. M., Mangili E. M., Simões, P. W., Ceretta, L. B., Tuon L. (2015). Sobrecarga no cuidado, estresse e impacto na qualidade de vida de cuidadores domiciliares assistidos na atenção básica. Cad. Saúde Colet. 23(2), 140-149.

Stackfleth, R., Diniz, M. A., Fhon, J. R. S., Vendruscolo, T. R. P., Fabrício-Whebe, S. C. C., Marques, S., \& Rodrigues, R. A. P. (2012). Sobrecarga de trabalho em cuidadores de idosos fragilizados que vivem no domicílio. Acta Paulista de Enfermagem, 25, 768-774.

Valeberg, B. T., \& Grov, E. K. (2013). Symptoms in the cancer patient-of importance for their caregivers' quality of life and mental health? European journal of oncology nursing, 17(1), 46-51.

Valentini, I. B., Zimmermann, N., \& Fonseca, R. P. (2010). Ocorrência de depressão e ansiedade em cuidadores primários de indivíduos com demência tipo Alzheimer: estudos de casos. Estudos interdisciplinares sobre o envelhecimento, 15(2). 